\title{
BRAF V600E Mutation Analysis
}

National Cancer Institute

\section{Source}

National Cancer Institute. BRAF V600E Mutation Analysis. NCI Thesaurus. Code C80273.

A molecular diagnostic test performed to determine the presence or absence of a BRAF V600E mutation in a tissue sample. 